\title{
Energetic Particles in Halos of Star Forming Galaxies
}

\author{
Yoel Rephaeli ${ }^{1,2 \star}$, Sharon Sadeh ${ }^{1}$ \\ ${ }^{1}$ School of Physics and Astronomy, Tel Aviv University, Tel Aviv, 69978, Israel \\ ${ }^{2}$ Center for Astrophysics and Space Sciences, University of California, San Diego, La Jolla, CA 92093-0424
}

Accepted XXX. Received YYY; in original form ZZZ

\begin{abstract}
Quantitative modeling of the spectro-spatial distributions of energetic electrons and protons in galactic halos is needed in order to determine their interactions with the local plasma and radiation fields, and also to estimate their residual spectral densities in intracluster and intergalactic environments. We develop a semi-analytic approach for calculating the particle distributions in the halo based on a detailed diffusion model for particle propagation from acceleration sites and interactions in the galactic disk. Important overall normalization of our models is based on results from detailed modeling in the Galactic disk with the GALPROP code. This provides the essential input for determining particle distributions in the outer disk, which are used as source terms for calculating the distributions in the extensive halo for a range of values of key parameters affecting energy losses and propagation mode. Our modeling approach is applied to the two edge-on star-forming galaxies NGC 4631 and NGC 4666, for which recent mapping of radio emission in the inner halo provides the required overall normalization. We predict the levels and spatial profiles of radio, X-ray, and $\gamma$-ray emission in the halos of these galaxies. Our quantitative modeling enables us to estimate the total calorimetric efficiencies of electrons and protons in star-forming galaxies, and to predict their residual spectral distributions in the outer halo and intergalactic space.
\end{abstract}

Key words: cosmic rays - galaxies: haloes - radio continuum: galaxies

\section{INTRODUCTION}

Measurements of energetic particles ('cosmic rays'), their radiative yields, and of magnetic fields in various media in interstellar space of the Galaxy have led to reasonably good knowledge of the particle spectro-spatial distributions (SSDs) in the Galactic disk, where the stellar particle acceleration sources are located. Particle propagation modes and energy loss processes are sufficiently well understood to expect that energetic electrons lose a large fraction of their energy by the time they reach the outer disk. This fraction is much smaller for protons whose (effective) energy loss timescale is much longer than a typical escape time from the disk. The distributions of both particles in the Galactic halo are of appreciable interest as they provide an essential basis for estimating particle escape rates from star-forming (SF) and starburst (SB) galaxies, and for quantifying their radiative yields in galactic halos (and IG space).

Outside the galactic disk, the couplings of protons and electrons to the lower density gas, weaker magnetic fields, and less intense radiation fields, are reduced but are expected to be still significantly stronger than in IG space. There has been little quantitative modeling of energetic particles in galactic halos, in spite of the fact that determining the particle distributions in these extended regions is needed for estimating their contributions to the mean IG spectral density. Additionally, the radiative yields in halos have to be estimated for separating these out from their respective values in the disk to enable precise determination of the latter, quite likely dominant emission. With increasing knowledge of the astrophysical environment in galactic halos, there is sufficient motivation for a more complete assessment of galactic energetic particles and their full emission in the disk and halo.

The radiative yields of electrons (by bremsstrahlung, Compton, and synchrotron processes) and protons, whose interactions with ambient protons produce neutral $\left(\pi^{0}\right)$ and charged $\left(\pi^{ \pm}\right)$pions which decay into $\gamma$-ray photons, electrons and

^ E-mail: yoelr@tauex.tau.ac.il 
positrons, respectively, cover the wide spectral range from radio to $\gamma$-ray. Measurement of this emission can potentially provide sufficient quantitative basis for determining the particle SSDs in the Galactic halo, in the halos of other SFGs, and in SBGs.

Modeling proton and electron spectral and spatial properties in the halo requires detailed knowledge of their SSDs in the disk. While semi-analytic calculations of these distributions have been made, mostly based on diffusion models (e.g., Syrovatskii 1959), currently the most realistic description of particle propagation in the Galactic disk is provided by the GALPROP code with which several diffusion (with and without re-acceleration) models were explored (Strong et al. 2010). For various purposes, it is useful to have a semi-analytic alternative to this code that can be easily employed to study predictions of various models for the spatial profiles of the gas density and magnetic field in the outer disk and halo, where the magnetized fully ionized gas properties and particle propagation modes are considerably simpler to model than in the inner much more complex disk.

A complete description of energetic particles in disk and inner halo of a SF or SB galaxy will provide the basis for determining their distributions in the outer halo and for estimating their contributions to the overall particle energy densities in IG space. For this purpose, an efficient, easily adjustable semi-analytic calculation scheme of energetic particle contributions by SF galaxies of various classes is of much interest in the context of a statistical study of the more general open issue of the origin and IG propagation of energetic particles. Results of such a study will also have important ramifications for understanding the origin of energetic electrons whose large-scale radio ('halo') emission has been mapped in many galaxy clusters. For example, the number of SFGs in a rich cluster may be sufficiently large for these to constitute an appreciable fraction of the emitting electrons in radio 'halos' (Rephaeli \& Sadeh 2016).

The motivation for detailed modeling of proton and electron SSDs in the outer disk and halo of a SF galaxy has recently been strengthened by radio measurements of the emission in the outer disks and inner halos of a sample of 35 nearby edge-on SFGs by the EVLA survey CHANG-ES (Wiegert et al. 2015). Analysis of these measurements yielded the first co-added median map of the inner halo emission of a spiral galaxy at $1.5 \mathrm{GHz}$. This map of the emission profile well beyond the galactic disk provides a quantitative basis for a reliable normalization of the SSD of primary and secondary electrons in the galactic halo, and for a realistic assessment of the predicted emission in the X-ray and $\gamma$-ray regions.

Here we describe proton and electron SSDs in the halo of a SFG based on a diffusion model for their propagation in the disk and halo as they traverse the magnetized gas and lose energy by all the relevant processes. In Section 2 we present the basic model and results of its implementation to the two nearby SFGs NGC 4631 and NGC 4666 for which radio emission has been measured in the inner halo. Our quantitative treatment provides a reliable basis for predicting the levels of halo radio, $\mathrm{X}-\&-\gamma$-ray emission in these galaxies, as detailed in Section 3, and further discussed in Section 4.

\section{PARTICLE DISTRIBUTIONS AND RADIATIVE YIELDS}

Particle acceleration in SFGs is largely a stellar-related phenomenon driven by shocks in SN remnants and pulsar wind nebulae, with the sources distributed across the stellar disk. The particles lose energy by interactions with the magnetized IS gas and radiation fields as they diffuse out of the acceleration sites across the disk, eventually reaching the outer disk and thereafter continue their (faster) random walk in the dilute halo. Extensive semi-analytic and numerical modeling of the particle propagation, energy losses, and their steady-state SSDs has focused on the complex disk environment where consequences of their interactions with IS media have either been measured (e.g., radio emission) or indirectly deduced (e.g., gas ionization and heating). Contrasting detailed models for the particle radiative yields with observations provides the basis for determining the particle distributions across the entire disk.

Current knowledge of particle distributions in galactic halos is poor, limited as it is to rough estimates based on qualitative considerations (based mainly on estimates of particle 'residence times' in the disk and 'calorimetric' fractions). A quantitatively reliable determination of the particle SSDs in the outer disk provides the essential input needed in the calculation of the distributions in the halo, and for (e.g.) estimating the residual particle spectral flux in the outer halo. Extension of the quantitative treatment of particle distributions to the halo by using detailed results of the distributions in the outer disk is a basic objective of our work.

Clearly, a detailed treatment of the inherently complex IS environment traversed by energetic particles, and the wide ranges of all the relevant quantities that affect their energy losses and propagation modes, necessitate an extensive numerical code to determine the particle SSDs. This provided the main motivation for developing the GALPROP code (e.g., Strong et al. 2010) ${ }^{1}$, currently the most comprehensive program which is widely used in modeling non-thermal (NT) phenomena in the Galaxy. The code is based on a solution to the kinetic equation for the particle steady-state distributions, asymptotically attained following diffusion of the particles from their acceleration sites into the full disk while losing energy by the various processes (and possibly also gaining energy by re-acceleration).

Because our main objective is a reasonably realistic modeling of proton and electron distributions and their predicted

1 The current version of GALPROP is available at https://gitlab.mpcdf.mpg.de/aws/galprop 
radiative yields in the galactic halo, a more optimal approach for our purposes is a simpler semi-analytic treatment that can be easily implemented in comparison with the use of a more detailed (but cumbersome) full-fledged code. However, we do use detailed results from extensive GALPROP modeling of the Galaxy in order to normalize our models to ensure consistency with observational data. The most relevant GALPROP-based study is that of Strong et al. (2010), who explored several diffusion models for energetic proton and electron spectral distributions in the Galactic disk, and determined their bolometric radio, X-\& - $\gamma$-ray yields. (For more recent GALPROP-based studies of the Galaxy, see Orlando \& Strong 2013, and Orlando 2019.) As we describe below, we consider similar models to those explored by Strong et al. (2010), and adopt the same initial proton and electron power-law spectra and source distribution (which is based on the observed pulsar population).

The above modeling approach is applied next to the two SFGs NGC 4631 and NGC 4666, for which there are no observationally-normalized GALPROP models for comparison. However, the radio intensity maps for the disk and inner halo (Wiegert et al. 2015) - the main motivation for our selection of these nearby edge-on galaxies for this study - provide the basic normalization of our models. Other available observationally determined model parameters for each galaxy are the SF rate, mean magnetic field in the disk, and luminosities in the visible and FIR bands.

\subsection{Particle Distributions}

The spatial distribution of particle sources (e.g., SNR, pulsar wind nebulae) can be modeled by a Gaussian (e.g., Syrovatskii 1959) which, for an ellipsoidal disk morphology with semi-major and semi-minor axes $a$ and $b$, is

$Q\left(\overrightarrow{r_{i}}\right)=\frac{1}{\pi^{3 / 2}} \frac{1}{a^{2} b} e^{-\frac{x_{i}^{2}+y_{i}^{2}}{a^{2}}-\frac{z_{i}^{2}}{b^{2}}}$,

where $\overrightarrow{r_{i}} \equiv\left(x_{i}, y_{i}, z_{i}\right)$ is the position vector of source $i$ in Cartesian coordinates.

The SSD of primary electrons at the post-diffusion position vector $\overrightarrow{r_{o}}$ and time $t_{o}$ is the Green function solution of the diffusion equation (Atoyan, Aharonian, \& Völker 1995):

$f_{e}\left(\overrightarrow{r_{o}}, t_{o}, \gamma\right)=\int_{0}^{t_{o}} \frac{\Delta \dot{N}_{e}\left(\gamma_{t}\right) P_{e}\left(\gamma_{t}\right)}{\pi^{3 / 2} P_{e}(\gamma) r_{d}^{3}} e^{-\frac{r^{2}}{r_{d}^{2}}} d t_{i}$

where $r \equiv\left|\overrightarrow{r_{o}}\right|, \gamma$ and $\gamma_{t}$ are, respectively, the post-and pre-diffusion energies of the electron, $\dot{N}_{e}\left(\gamma_{t}\right)$ is the spectrum of the source-injected electrons, $\mathrm{P}_{e}(\gamma)$ is the energy loss rate, and $r_{d}$ is the diffusion radius. Combining equations (1) and (2) as

$$
f_{e}\left(\overrightarrow{r_{o}}, t_{o}, \gamma\right)=\frac{1}{\pi^{3 / 2}} \frac{1}{a^{2} b} \int_{0}^{t_{o}} d t_{i} \frac{\Delta \dot{N}_{e}\left(\gamma_{t}\right) P_{e}\left(\gamma_{t}\right)}{\pi^{3 / 2} P_{e}(\gamma) r_{d}^{3}} \iiint_{V} e^{-\frac{\left(x_{o}-x_{i}\right)^{2}+\left(y_{o}-y_{i}\right)^{2}}{r_{d}^{2}}-\frac{\left(z_{o}-z_{i}\right)^{2}}{r_{d}^{2}}} e^{-\frac{x_{i}^{2}+y_{i}^{2}}{a^{2}}-\frac{z_{i}^{2}}{b^{2}}} d x_{i} d y_{i} d z_{i},
$$

yields the total post-diffusion distribution of primary electrons at $\overrightarrow{r_{o}}$ and time $t_{o}$. With the steeply varying Gaussian source distribution, the volume-integration limits can be changed to infinity without appreciable loss of accuracy; doing so results in

$$
f_{e}\left(\overrightarrow{r_{o}}, t_{o}, \gamma\right)=\frac{1}{\pi^{3 / 2}} \frac{\Delta \dot{N}_{e}\left(\gamma_{t}\right) P_{e}\left(\gamma_{t}\right)}{P_{e}(\gamma)\left(a^{2}+r_{d}^{2}\right) \sqrt{b^{2}+r_{d}^{2}}} e^{-\frac{x_{o}^{2}+y_{o}^{2}}{a^{2}+r_{d}^{2}}-\frac{z_{o}^{2}}{b^{2}+r_{d}^{2}}}
$$

The starting point for calculation of the secondary electron SSD is the equivalent solution for source-injected protons, which describes the distribution of post-diffusion protons just before they interact with protons in the ambient gas. Secondary electrons and positrons are produced in these proton-proton interactions via muon decays (following their production in charged pion decays), at position $\overrightarrow{r_{m}}$ and time $\mathrm{t}_{m}$

$$
f_{p}\left(r_{m}, t_{m}, \gamma\right)=\frac{1}{\pi^{3 / 2}} \frac{\Delta \dot{N}_{p}\left(\gamma_{t}\right) P_{p}\left(\gamma_{t}\right)}{P_{p}(\gamma)\left(a^{2}+r_{d}^{2}\right) \sqrt{b^{2}+r_{d}^{2}}} e^{-\frac{x_{m}^{2}+y_{m}^{2}}{a^{2}+r_{d}^{2}}-\frac{z_{m}^{2}}{b^{2}+r_{d}^{2}}}
$$

The SSD of post-diffusion secondary electrons can be derived by converting the diffused proton distribution into a distribution of pre-diffusion electrons, and treating the latter as the source for diffusion of secondary electrons. Since electrons in the galactic disk lose essentially all (as is quantified in the next section) their energy before diffusing to the inner halo, it can be assumed that only the source electrons in the halo contribute to the population of the post-diffusion secondary electrons. Therefore, the spatial integrals over $\vec{m}$ can be performed in the $[-\infty, \infty]$ range, resulting in the expression

$$
\begin{aligned}
f_{e}\left(\overrightarrow{r_{o}}, t_{o}, \gamma\right)= & \frac{1}{\pi^{3 / 2}} \frac{1}{A} \frac{16}{3} c n N_{0 p} \sigma_{p p}^{\pi^{0}, \pi^{ \pm}} \times 0.14 \frac{1}{P_{e}(\gamma)} \int_{t_{m}}^{t_{o}} d t_{m}\left(0.56 \frac{\gamma_{t}}{A}\right)^{-\alpha_{p}} P\left(\gamma_{t}\right) \\
& \int_{0}^{t_{m}} d t_{i} \frac{e^{\ell_{p}\left(t_{m}-t_{i}\right)\left(1-\alpha_{p}\right)} e^{-\frac{x_{o}^{2}+y_{o}^{2}}{a^{2}+4 D\left(t_{m}-t_{i}\right)+r_{d}^{2}}-\frac{z_{o}^{2}}{a^{2}+4 D\left(t_{m}-t_{i}\right)+r_{d}^{2}}}}{\left.\left[a^{2}+4 t_{m}-t_{i}\right)+r_{d}^{2}\right] \sqrt{b^{2}+4 D\left(t_{m}-t_{i}\right)+r_{d}^{2}}}
\end{aligned}
$$

where $A=(1 / 4)\left(m_{\pi} / m_{e}\right) \approx 70$ (Ramaty \& Lingenfelter 1966), $n$ is the gas number density, $N_{o p}$, and $\alpha_{p}$ are the coefficient and power index of the proton injection spectrum, $\sigma_{p p}^{\pi^{0}, \pi^{ \pm}} \approx 35 \mathrm{mb}$ (obtained by averaging over an analytical approximation for the cross section of p-p interactions obtained for proton energies in the range $0.01-100 \mathrm{GeV}$ [Kelner et al. 2006]), is the mean cross section for p-p interactions, $\ell_{p} \equiv n c \kappa \sigma_{p p}^{\pi^{0}, \pi^{ \pm}}$, and $\kappa \approx 0.45$, is the inelasticity of p-p interactions (Aharonian \& Atoyan 1996). 
Energy loss processes for energetic electrons are electronic excitations (in Coulomb scattering), bremsstrahlung, and Compton-synchrotron, with the total energy loss rate given by

$P(\gamma)=p_{0}+p_{1} \gamma+p_{2} \gamma^{2}$

where $p_{0} \simeq 1.1 \times 10^{-15}\left(n_{e} / 10^{-3} \mathrm{~cm}^{-3}\right) \mathrm{s}^{-1}$ is the loss rate by electronic excitations in fully ionized gas (Gould 1972); $n_{e}$ is the gas electron density. The coefficients of the bremsstrahlung and Compton-synchrotron rates (e.g., Blumenthal \& Gould 1970) are $p_{1} \simeq 1.3 \times 10^{-18} \times\left(n_{e} / 10^{-3} \mathrm{~cm}^{-3}\right) \mathrm{s}^{-1}$, and $p_{2}=s_{0}\left[(1+z)^{4}+0.1 \times\left(B /\left(10^{-6} \mu G\right)\right)^{2}\right] \mathrm{s}^{-1}$, respectively, with $s_{0}=1.3 \times 10^{-20} \mathrm{~s}^{-1}$ in the galactic halo, where Compton scattering is largely off the CMB (whose energy density increases steeply with redshift), unlike in the galactic disk where the stellar radiation field is stronger. The main energy loss of energetic protons is by interactions with gas protons at a rate $p\left(\gamma_{p}\right)=n_{p}(r) c \kappa \sigma_{p p}^{\pi^{0}, \pi^{ \pm}} \gamma_{p}$, where $\sigma_{p p}^{i n}$ is the mean cross section in inelastic scatterings.

\subsubsection{Disk Distributions}

In our approximate analytical calculations of proton and electron SSDs in the disk we use mean values of the gas density, magnetic field, and diffusion coefficient; doing so facilitates the computation of the radiative yields (e.g., emissivities). The primary electron SSD is then

$$
\begin{aligned}
f_{e}\left(t_{o}, \gamma\right) & =\int_{0}^{t_{o}} \frac{1}{\pi^{3 / 2}} \frac{\Delta \dot{N}\left(\gamma_{t}\right) P\left(\gamma_{t}\right)}{P(\gamma)\left(a^{2}+r_{d}^{2}\right) \sqrt{b^{2}+r_{d}^{2}}}\left[2 \int_{0}^{b} d z_{o} \int_{0}^{a \sqrt{1-z_{o}^{2} / b^{2}}} 2 \pi x_{o} d x_{o}\right] e^{-\frac{x_{o}^{2}}{a^{2}+r_{d}^{2}}-\frac{z_{o}^{2}}{b^{2}+r_{d}^{2}}} \\
& =\int_{\gamma_{\min }}^{\gamma_{\max }} \frac{d \gamma}{P(\gamma)} \int_{t_{\text {min }}}^{t_{o}} \Delta \dot{N}\left(\gamma_{t}\right) P\left(\gamma_{t}\right) \\
& \times\left[\operatorname{erf}\left(\frac{b}{\sqrt{b^{2}+r_{d}^{2}}}\right)-\frac{b}{\sqrt{b^{2}+r_{d}^{2}}} e^{-a^{2} /\left(a^{2}+r_{d}^{2}\right)} \frac{\operatorname{erf}\left(r_{d} \sqrt{1 /\left(a^{2}+r_{d}^{2}\right)-1 /\left(b^{2}+r_{d}^{2}\right)}\right)}{r_{d} \sqrt{1 /\left(a^{2}+r_{d}^{2}\right)-1 /\left(b^{2}+r_{d}^{2}\right)}}\right]
\end{aligned}
$$

The corresponding expression for secondary electrons is

$$
\begin{aligned}
f_{e}\left(t_{o}, \gamma\right)= & \frac{1}{A} \frac{16}{3} c n N_{o p} \sigma_{p p}^{\pi^{0}, \pi^{ \pm}} \times 0.13957 \int_{\gamma} \frac{d \gamma}{P_{e}(\gamma)} \int_{t_{m}}^{t_{o}} d t_{m}\left(0.558281 \frac{\gamma_{t}}{A}\right)^{-\alpha_{p}} P\left(\gamma_{t}\right) \\
& \int_{0}^{t_{m}} d t_{i} \frac{e^{\ell_{p}\left(t_{m}-t_{i}\right)\left(1-\alpha_{p}\right)}}{\sqrt{b^{2}+4 D\left(t_{m}-t_{i}\right)+r_{d}^{2}}}\left[\sqrt{b^{2}+4 D\left(t_{m}-t_{i}\right)+r_{d}^{2}} \operatorname{erf}\left(\frac{b}{\sqrt{b^{2}+4 D\left(t_{m}-t_{i}\right)+r_{d}^{2}}}\right)\right. \\
& \left.-b e^{-a^{2} /\left[a^{2}+4 D\left(t_{m}-t_{i}\right)+r_{d}^{2}\right]} \frac{\operatorname{erfi} \sqrt{a^{2} /\left[a^{2}+4 D\left(t_{m}-t_{i}\right)+r_{d}^{2}\right]}}{\sqrt{a^{2} /\left[a^{2}+4 D\left(t_{m}-t_{i}\right)+r_{d}^{2}\right]}}\right],
\end{aligned}
$$

where erfi is the imaginary error function.

The galactic disk is modeled as an oblate ellipsoid with semi-major and semi-minor axes measuring $20 \mathrm{kpc}$ and $4 \mathrm{kpc}$, respectively. Mean values of the magnetic field in the disks of NGC 4631 and NGC 4666 were observationally estimated to be 10 and $14 \mu \mathrm{G}$, respectively (Stein 2017). The assumed disk mean gas density, $n=0.3 \mathrm{~cm}^{-3}$, corresponds to a total (disk) gas mass of approximately $3 \times 10^{10}$ solar masses. Finally, the diffusion coefficient D is set to $3.4 \times 10^{28} \mathrm{~cm}^{2} \mathrm{~s}^{-1}$, in accord with the value taken by Strong et al. in their plain diffusion model 2. The radio luminosity generated by the disk at $1.575 \mathrm{GHz}$, as well as the hard X-ray (10 keV-200 keV), and $\gamma(100 \mathrm{MeV}-100 \mathrm{GeV})$ luminosities, are calculated by integrating the respective emissivities over the spectral distribution functions of primary and secondary electrons, and neutral pions, with the latter derived from the corresponding proton distribution function, Eq. (18).

\subsubsection{Halo Distributions}

As we noted, our description of energetic protons and electrons in the disk, which is similar to the more detailed work of Strong et al. (2010), provides the basis for the first quantitative modeling of the proton and electron distributions in the halo. We adopt the commonly used $\beta$-King model to describe the spatial profile of the gas density in the halo selecting values of the core radius and the index $\beta$ in the observationally deduced ranges, with the central density as a dependent parameter whose value is determined from the total gas mass. The magnetic field profile in the halo is related to that of the gas density by assuming conservation of magnetic flux - appropriate in a highly electrically conductive, fully ionized halo gas - implying the scaling $\sim n^{2 / 3}$.

We divide the halo (excluding the galactic disk) into two separate regions: an inner cylindrical region with a radius of 20 $\mathrm{kpc}$ and height that extends from $\mathrm{r}_{z}=4 \mathrm{kpc}$ to $20 \mathrm{kpc}$. The $\beta$-King model is specified by the halo parameters (Table 1), with the $\mathrm{z}$-dependence of the magnetic field and density profiles given by $\left[1+\left(r_{z} / r_{c}\right)^{2}\right]^{-3 \beta / 2}$. The outer halo region (at $r \geq 20$ $\mathrm{kpc})$ is taken to be spherical with a density profile $\left[1+\left(r / r_{c}\right)^{2}\right]^{-3 \beta / 2}$.

Based on (rough) estimates of the mean strength of the IG field, we set a lower limit of $30 \mathrm{nG}$ on its value, which is 
relevant only in the outer halo region. These analytic distributions allow the construction of 3-dimensional emissivity grids, thereby significantly simplifying integrations along the los and convolution with a Gaussian beam to produce radio brightness profiles and volume-integrated emissivities to compute total luminosities in the radio, hard X-ray, and $\gamma$ bands. The emissivity grid for the halo extends from the outer surface of the (model) disk out to $x_{o}=y_{o}=z_{o}=200 \mathrm{kpc}$.

\subsection{Particle Radiative Yields}

The radiative yields of primary electrons, protons, and secondary electrons (and positrons) can be computed by integrating the relevant radiation emissivities over the particle distributions detailed in the previous subsection. Accordingly, the spectral radio emissivity due to a population of relativistic electrons is (Blumenthal \& Gould 1970)

$\frac{d E}{d \nu d t}=\frac{\sqrt{3} k e^{3} B}{4 \pi m_{e} c^{2}} \int d \Omega_{\alpha} N_{e} \sin \alpha \times \int_{\gamma_{1}}^{\gamma_{2}} f\left(\overrightarrow{r_{o}}, t_{o}, \gamma\right) d \gamma \frac{\nu}{\nu_{c}} \int_{\nu / \nu_{c}}^{\infty} K_{5 / 3}(\xi) d \xi$

where the electron density $N_{e}$ generally depends also on the pitch angle, $\alpha, \gamma_{1}$ and $\gamma_{2}$ bracket the electron spectral distribution, $\nu_{c}$ is the cyclotron frequency, $K_{5 / 3}$ is the modified Bessel function of $5 / 3$ order, and the physical constants have their usual meaning.

The (photon) emissivity due to Compton scattering of low-energy CMB photons off relativistic electrons derived in the Thomson limit is (Blumenthal \& Gould 1970)

$\frac{d N_{\gamma, \epsilon}}{d \epsilon_{1} d t}=\frac{\pi r_{0}^{2} c}{2 \gamma^{4}} \frac{n(\epsilon) d \epsilon}{\epsilon^{2}}\left(2 \epsilon_{1} \log \frac{\epsilon_{1}}{4 \gamma^{2} \epsilon}+\epsilon_{1}+4 \gamma^{2} \epsilon-\frac{\epsilon_{1}^{2}}{2 \gamma^{2} \epsilon}\right)$,

where $\epsilon$ and $\epsilon_{1}$ are the pre- and post-scattering photon energies, respectively. The electron classical radius is $r_{0} \equiv e^{2} / m_{e} c^{2}$, and $n(\epsilon)$ is the spectral energy density of the scattering radiation fields in the disk, consisting, in addition to the CMB, of diluted Planckian distributions at temperatures 3500K (starlight), and 30K (dust). Some $70 \%$ of the Galactic stellar radiation field originates in a 'thin' disk and the rest in a 'thick' disk, with the former modeled as an ellipsoid with semi-major and semi-minor axes of $10 \mathrm{kpc}$ and 300 pc (e.g., Gilmore \& Reid 1983), and the latter is the remaining disk volume.

By equating the stellar luminosity (in either the optical or IR bands) with the surface-integrated flux for an isotropic diluted blackbody, $F=4 \delta \sigma_{b} T^{4}$, where $\delta$ is the dilution factor and $\sigma_{b}$ is the Stefan-Boltzmann constant, the respective dilution factors can be computed for the two disk regions. The dilution factors for the stellar and dust fields are determined based on the estimate that of the total Galactic stellar luminosity, $\sim 5 \times 10^{10} \mathrm{~L}_{\odot}$ (Strong et al. 2010 ), $20 \%$ is processed into the IR by interstellar dust. The Compton energy loss rate in the stellar radiation field is

$\frac{d E}{d t}=\frac{4}{3} \sigma_{T} c \frac{4 \delta \sigma_{b} T^{4}}{c} \gamma^{2}$

where $\sigma_{T}$ is the Thomson cross section. Integrating over the energy $\epsilon$ and the scattering electron population yields the total Compton emissivity

$\frac{d E_{t o t}\left(\epsilon_{1}, \overrightarrow{r_{o}}\right)}{d \epsilon_{1} d t}=\int_{\gamma_{1}}^{\gamma_{2}} \int_{\epsilon_{1} / 4 \gamma^{2}}^{\infty} \frac{d N_{\gamma, \epsilon}}{d \epsilon_{1} d t} \epsilon \times f\left(\overrightarrow{r_{o}}, t_{o}, \gamma\right) d \epsilon d \gamma$

The spectral $\gamma$-ray emission generated by $\pi^{0}$ decay can be calculated as (Mannheim \& Schlickeiser 1994):

$f_{\gamma}\left(E_{\gamma}, \overrightarrow{r_{o}}\right)=2 E_{\gamma} \int_{\left.E_{\gamma}+\left[\left(m_{\pi^{0}} c^{2}\right)^{2} / 4 E_{\gamma}\right)\right]}^{\infty} \frac{f_{\pi^{0}}\left(E_{\pi^{0}}, \overrightarrow{r_{o}}\right) d E_{\pi^{0}}}{\sqrt{E_{\pi^{0}}^{2}-m_{\pi^{0}}^{2} c^{4}}}$,

where (Pfrommer \& Enßlin 2004)

$f_{\pi^{0}}\left(E_{\pi^{0}}, \overrightarrow{r_{o}}\right)=8 \mathrm{cnf} f_{p}\left(\overrightarrow{r_{o}}, t_{o}, \gamma\right) \sigma_{p p}^{\pi^{0}, \pi^{ \pm}}\left(\frac{6 E_{\pi^{ \pm}}}{G e V}\right)^{-\frac{4}{3}\left(\alpha-\frac{1}{2}\right)} G e V^{-1}$.

\subsection{Model Parameters}

As noted already, our approximate description of energetic proton and electron propagation, gas density and magnetic field distributions in the Galactic disk is chosen such that it is in general agreement with the more detailed GALPROP models of Strong et al. (2010). This is affected by selecting key parameter values that are reasonably close to those of the second diffusion model which is fully specified in the latter paper. We emphasize again that the main goal in our modeling of the disk is mostly to provide a realistic basis for determining proton and electron distributions and their yields in the Galactic halo where gas and magnetic field properties are poorly known, and can only be modeled by considering reasonable ranges of the key parameters.

As can be seen in table (1), we consider 4 galactic halo models, each of which is a variation of the 4 basic parameters $\left(r_{c}, \beta, \mathrm{n}, \mathrm{D}\right)$, where $M_{g}$ is the halo gas mass. We use a single model to characterize the disk, as discussed in the previous subsection. 


\begin{tabular}{|l|l|c|c|c|c|c|}
\cline { 3 - 7 } \multicolumn{2}{c|}{} & $\mathrm{r}_{c}(\mathrm{kpc})$ & $\beta$ & $\mathrm{n}\left(\mathrm{cm}^{-3}\right)$ & $\mathrm{D}\left(\mathrm{cm}^{2} \mathrm{~s}^{-1}\right)$ & $\mathrm{M}_{g}\left(\mathrm{M}_{\odot}\right)$ \\
\hline \multirow{4}{*}{ NGC 4631 } & model 1 & 2 & .85 & 1 & $3 \times 10^{30}$ & $3 \times 10^{10}$ \\
& model 2 & 3 & .85 & .4 & $3 \times 10^{30}$ & $3 \times 10^{10}$ \\
& model 3 & 2 & .85 & 1 & $6 \times 10^{30}$ & $3 \times 10^{10}$ \\
& model 4 & 2 & .7 & .25 & $3 \times 10^{30}$ & $3 \times 10^{10}$ \\
\hline \multirow{4}{*}{ NGC 4666 } & model 1 & 2 & 1.05 & 4 & $3 \times 10^{30}$ & $3 \times 10^{10}$ \\
& model 2 & 3 & 1.05 & 1.3 & $3 \times 10^{30}$ & $3 \times 10^{10}$ \\
& model 3 & 2 & 1.05 & 4 & $6 \times 10^{30}$ & $3 \times 10^{10}$ \\
& model 4 & 2 & .85 & 1.1 & $3 \times 10^{30}$ & $3 \times 10^{10}$ \\
\hline
\end{tabular}

Table 1. Model parameters
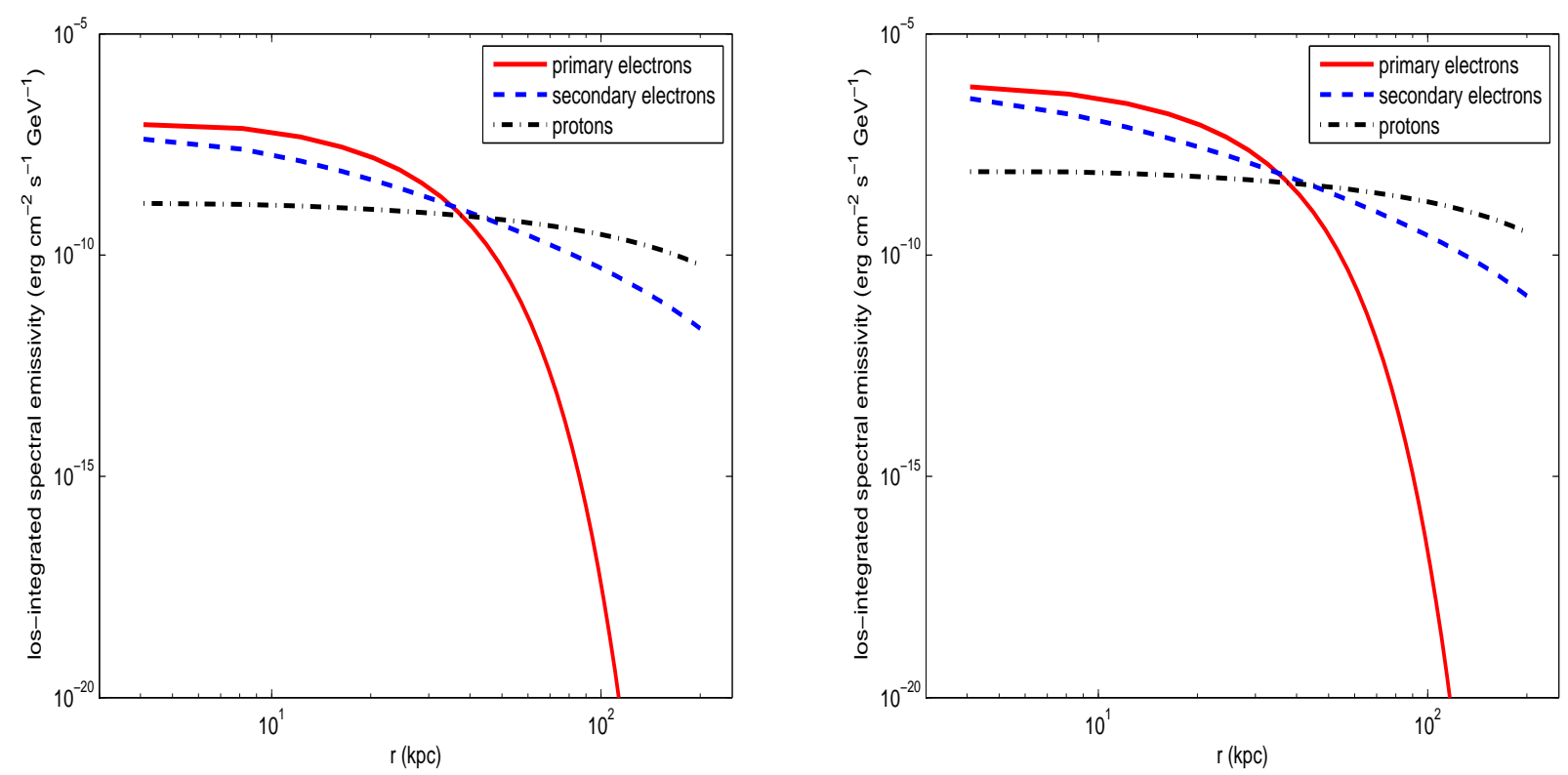

Figure 1. Time-averaged los-integrated particle surface emissivity profiles above the galactic plane, computed for $\gamma_{e}=10^{4}$ (primary and secondary electrons) and $\gamma_{p} \approx 12$ (protons). Results are shown for NGC 4631 (left) and NGC 4666 (right)

\section{RESULTS}

We applied our modeling approach to predict the levels and spatial profiles of proton and electron radiative yields in the Galactic halo and in the halos of the two star forming, edge-on galaxies NGC 4631 and NGC 4666 at the respective distances of 7.4 and $27.5 \mathrm{Mpc}$, and with star formation rates (SFRs) of 1.33 and $7.29 \mathrm{M}_{\odot} \mathrm{yr}^{-1}$ (Wiegert et al. 2015). The particle source distribution in the latter two galaxies was assumed to be as in the Galaxy, and the overall injection rates were determined by scaling up the Galactic rate, $\simeq 1 \mathrm{M}_{\odot} \mathrm{yr}^{-1}$, by the respective SFR of each galaxy. As shown in table (1), models $1-4$ differ only in the value of a single parameter, with the central gas number density adjusted so as to yield the same halo gas mass of $3 \times 10^{10} \mathrm{M}_{\odot}$.

The spatial profiles of primary and secondary electrons at $\gamma_{e}=10^{4}$ (contributing most of the synchrotron emission at $\nu=1.5 \mathrm{GHz}$ ), and protons at $\gamma_{p} \approx 12$ (kinetic energy of $\sim 10.3 \mathrm{GeV}$, above the threshold energy for pion production), are illustrated in Fig. (1); these are essentially Eqs. (4), (5), and (7), multiplied by the respective particle energy. The enhanced population of protons and secondary electrons above the galactic plane is clearly manifested, in clear contrast with the steeply falling profile of primary electrons.

For each galaxy we calculated the radio, hard X-ray, and $\gamma$ spectral luminosities and brightness profiles at $1.575 \mathrm{GHz}$, $20 \mathrm{keV}$, and $100 \mathrm{MeV}$, as well as spectra and bolometric luminosities in the 10-200 keV and $1 \mathrm{MeV}-100 \mathrm{GeV}$ bands. Radio brightness profiles (in mJy/beam) for the 4 models are plotted in Fig. (2) for NGC 4631 (left-) and NGC 4666 (right-hand panel). Intersections of the brightness contours measured by Wiegert et al. (2015) with a line perpendicular to the galactic plane and passing through its centre were read off the brightness map; for each contour above and below the plane angular distances of the intersection points were converted into physical distances and averaged. Measurement errors were estimated as the square root of the contour rms noise and thickness added in quadratures. Model predictions, smoothed with a Gaussian beam of $\sim 18$ " FWHM, were normalized either to the 3rd (NGC 4631) or 4th (NGC 4666) data point; these refer only to the halo, and consequently the two innermost measurements for NGC 4631, which still lie in the galactic disk, are irrelevant to 

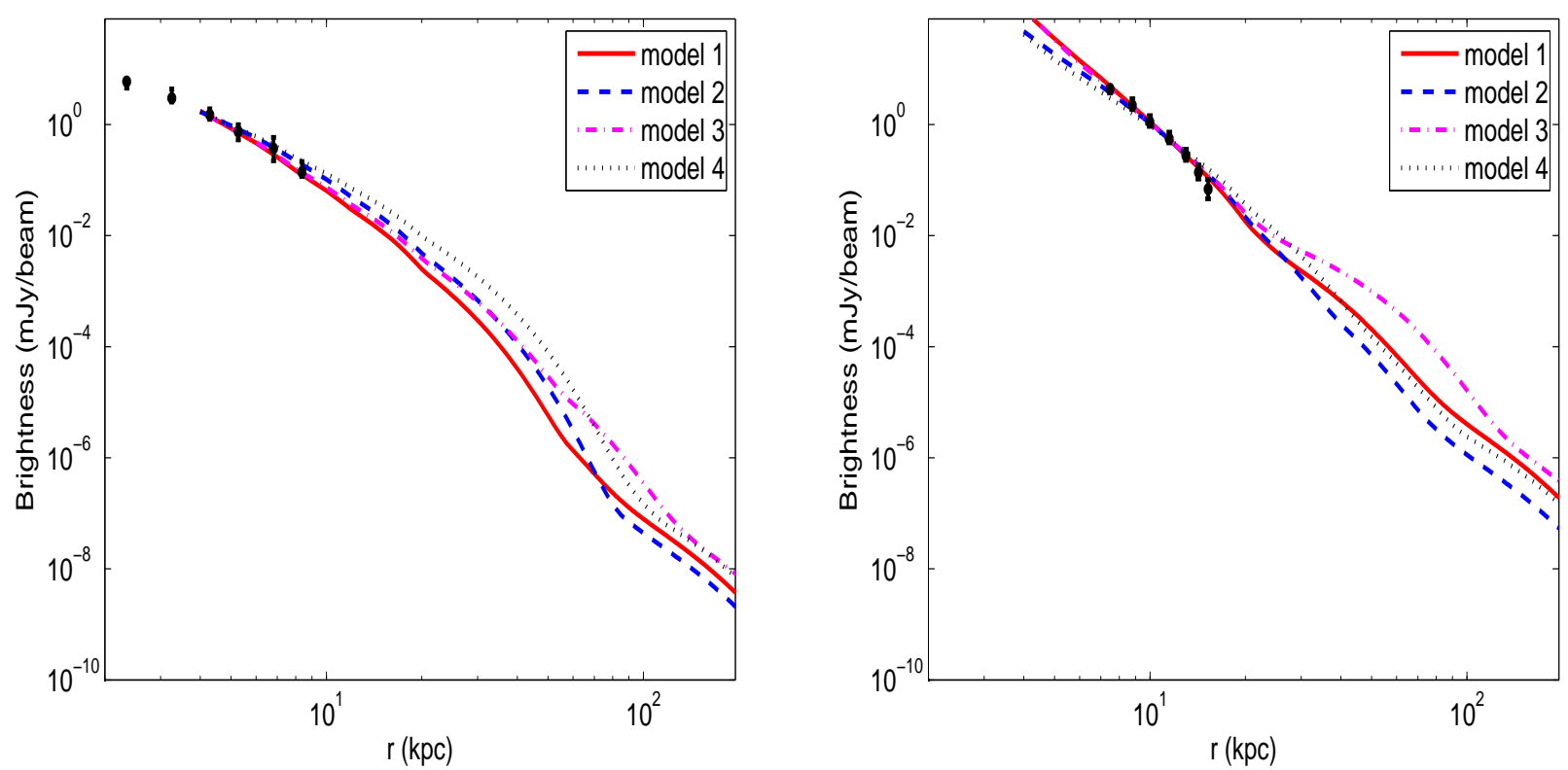

Figure 2. Radio-brightness profiles at $\nu=1.575 \mathrm{GHz}$, smoothed with a Gaussian beam of FWHM 18". Results are shown for NGC 4631 (left) and NGC 4666 (right). Parameter values are listed in table (1).

the halo. The change of slope apparent in the brightness profiles at $\sim 30-80 \mathrm{kpc}$ (depending on model) marks the height above (or below) the disk where the radio-yield of secondary electrons begins to dominate over that of primary electrons.

Keeping the same total halo gas mass with a higher core radius (model 2) and lower $\beta$ (model 4) implies lower central densities. This clearly results in reduced densities of secondary electrons, and consequently leads to the lower (with respect to model 1) radio brightness levels apparent in the secondary electron-dominated region of model 2; while the implied densities in model 4 are lower as well, the more moderate fall of the gas profile actually gives rise to a somewhat less steep brightness profiles, lying slightly above and below the model 1 profiles in the secondary-electron dominated zone for NGC 4631 and NGC 4666, respectively. By a qualitative comparison between the measurements and model predictions we infer that for NGC 4631 model 2 best matches the observations, whereas model 1 provides the closest fit for NGC 4666; these are referred to as "fiducial" models.

The los-integrated emissivity profile at $20 \mathrm{keV}$ and the hard X-ray spectrum in the 10-200 keV band (for the fiducial models) are illustrated in Fig. (3). The profile essentially reflects that of the electrons since it primarily results from electron scattering off the (uniform) CMB. For NGC 4666 models 1, 3, and 4 are roughly similar, whereas for NGC 4631 the profile calculated for model 2 joins the model 1 and 4 profiles at $\sim 40 \mathrm{kpc}$. The emissivity profile for model 3 reflects the faster diffusion from the inner to the outer halo, which is clearly due to the higher diffusion coefficient in this model. As expected, the spectral X-ray luminosity in the disk and halo, shown in the lower panels of the figure, is essentially a power-law in energy, with spectral indices in (the 10-100 keV band) of .93 and 1.00 (NGC 4631), and .98 and 1.01 (NGC 4666) respectively. More generally, since particle acceleration is directly related to SF, the higher luminosities of NGC 4666 exactly reflect the higher (by a factor of 5.33) SFR in this galaxy than that in NGC 4631.

The los-integrated emissivity profile at $100 \mathrm{MeV}$ and the $\gamma$-spectrum in the $1 \mathrm{MeV}-100 \mathrm{GeV}$ band are illustrated in Fig. (4) for the fiducial models. Up to $r \sim 40 \mathrm{kpc}(\mathrm{NGC} 4631)$ and $\sim 20 \mathrm{kpc}$ (NGC 4666) above the galactic plane, the highest emission levels are predicted for models 1 (high central gas density) and 2 (large core radius); at larger distances the milder density slope of model 4 results in the highest emission in both galaxies. Also, the relatively low emission levels in model 3 reflect the high diffusion coefficient, which results in faster proton diffusion out of the inner higher density region of the halo. As can be clearly seen in the lower panels of the figure, the fraction of the $\gamma$ emission that originates in the halo is negligible with respect to that from the disk, essentially due to the lower gas densities outside the galactic disk.

The radio flux at $1.575 \mathrm{GHz}$, hard X-ray luminosity in the $10-200 \mathrm{keV}$, and $\gamma$-ray luminosity above $100 \mathrm{MeV}$ are listed in table (2), where the disk, halo, and total contributions are explicitly specified. These results refer to the respective fiducial model of each galaxy: model 2 for NGC 4631 and model 1 for NGC 4666. As is apparent from the table, the halo produces a non-negligible emission only in the hard X-ray band, constituting some 93-95\% of the total luminosity of each galaxy.

Finally, we calculated the radiative efficiencies for particle energy conversion to the radio and $\gamma$-radiation channels, results of which are listed in table (3), and compared with the respective factors specified by Strong et al. (2010). 

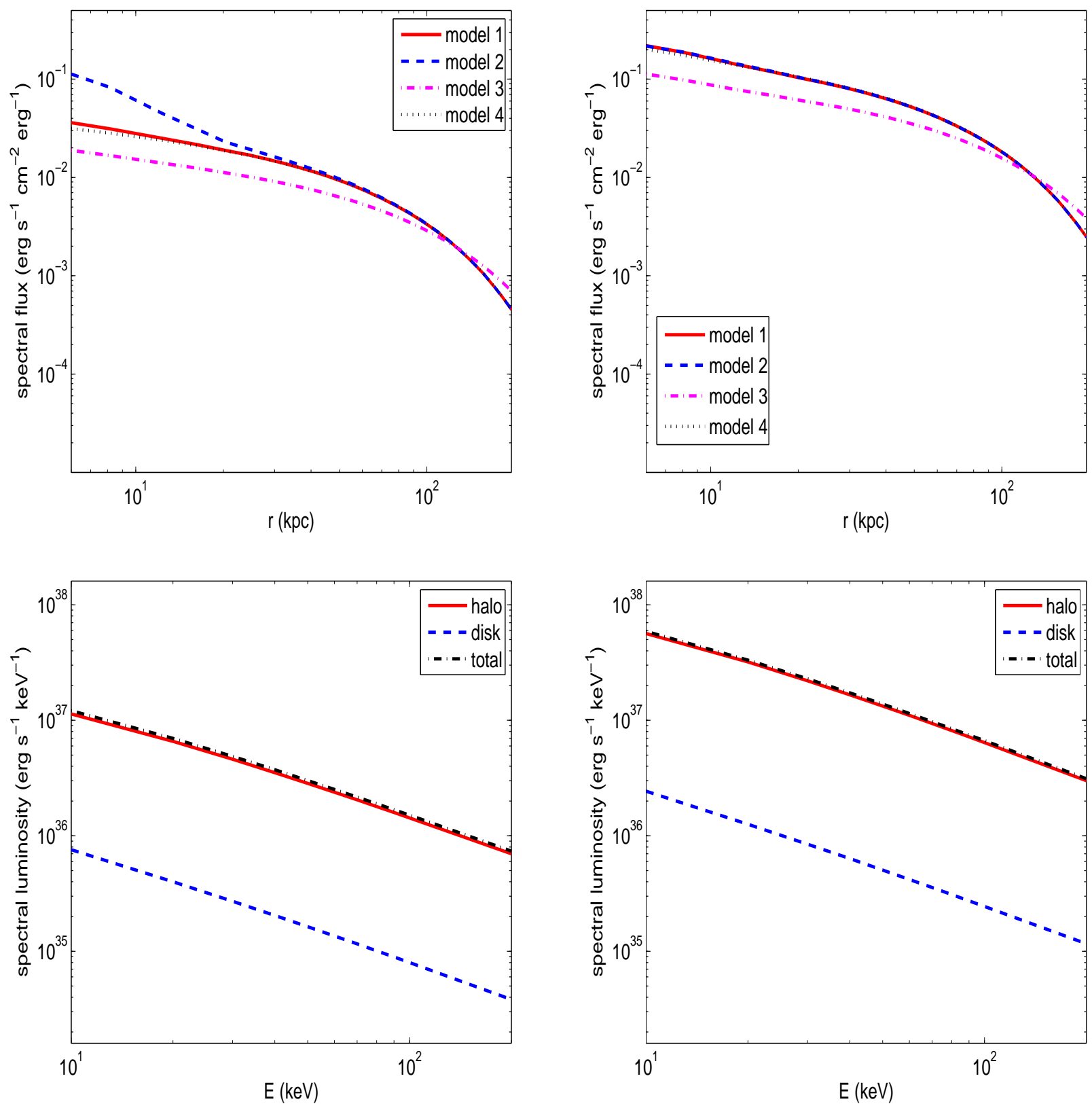

Figure 3. X-ray emission from NGC 4631 (left panels) and NGC 4666 (right panels). Upper panels: los-integrated hard X-ray emissivity profiles at $20 \mathrm{keV}$. Lower panels: 10-200 keV spectral luminosities of the disk and halo.

\begin{tabular}{|c|c|c|c|c|}
\cline { 3 - 5 } \multicolumn{2}{c|}{} & hard X-ray $(10-200 \mathrm{keV})$ & $\gamma(\geq 100 \mathrm{MeV})$ & $1.575 \mathrm{GHz}$ flux \\
\hline \multirow{3}{*}{ NGC 4631 } & disk & $2.5 \times 10^{37}$ & $3.2 \times 10^{39}$ & $695 \mathrm{mJy}$ \\
& halo & $4.2 \times 10^{38}$ & $3.4 \times 10^{38}$ & $36 \mathrm{mJy}$ \\
& total & $4.5 \times 10^{38}$ & $3.5 \times 10^{39}$ & $731 \mathrm{mJy}$ \\
\hline \multirow{3}{*}{ NGC 4666 } & disk & $7.8 \times 10^{37}$ & $1.8 \times 10^{40}$ & $306 \mathrm{mJy}$ \\
& halo & $2.0 \times 10^{39}$ & $3.3 \times 10^{39}$ & $3 \mathrm{mJy}$ \\
& total & $2.1 \times 10^{39}$ & $2.1 \times 10^{40}$ & $309 \mathrm{mJy}$ \\
\hline
\end{tabular}

Table 2. Computed radiative yields of NGC 4631 and NGC 4666. 

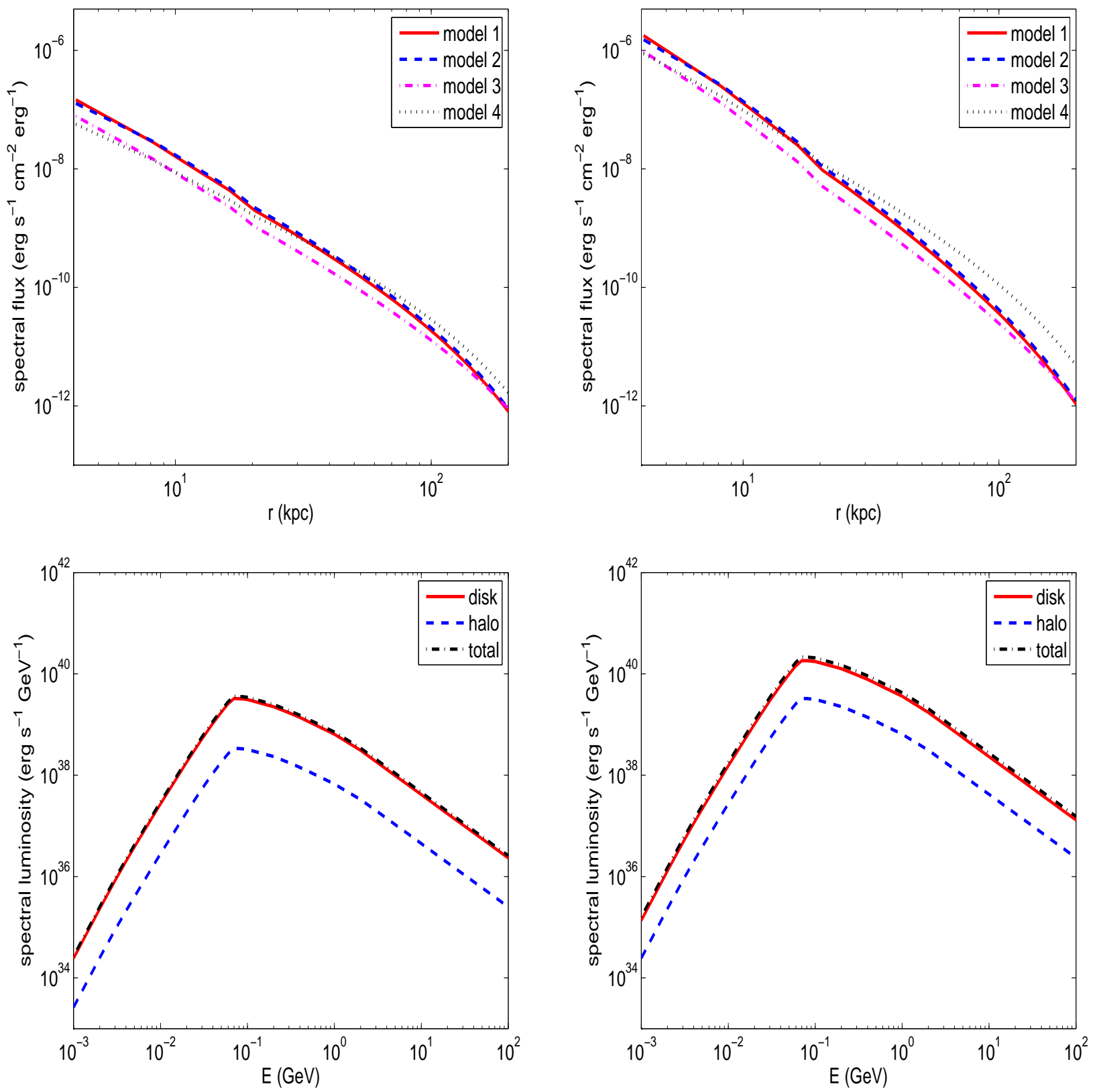

Figure 4. $\gamma$-ray emission from NGC 4631 (left panels) and NGC 4666 (right panels). Upper panels: profiles of the spectral flux at 100 $\mathrm{MeV}$. Lower panels: $1 \mathrm{MeV}-100 \mathrm{GeV}$ spectral luminosities in the disk and halo.

\begin{tabular}{|c|c|c|c|c|c|}
\cline { 3 - 6 } \multicolumn{1}{c|}{} & \multirow{2}{c|}{\begin{tabular}{c} 
Radio \\
\multicolumn{1}{c|}{}
\end{tabular}} & \multicolumn{2}{c|}{$\gamma:$ Compton } & \multicolumn{2}{c|}{$\gamma: \pi^{0}$-decay } \\
\cline { 3 - 6 } \multicolumn{1}{c|}{} & $.001-100 \mathrm{GHz})$ & $.01-100 \mathrm{MeV}$ & $100 \mathrm{MeV}-100 \mathrm{GeV}$ & $.01-100 \mathrm{MeV}$ & $100 \mathrm{MeV}-100 \mathrm{GeV}$ \\
\hline NGC 4631 & .08 & .20 & .17 & .002 & .04 \\
\hline NGC 4666 & .09 & .20 & .15 & .002 & .04 \\
\hline Milky Way & .16 & .14 & .14 & .0002 & .007 \\
\hline
\end{tabular}

Table 3. Radiative efficiencies for NGC 4631 and NGC 4666, compared with those determined by Strong et al. (2010) for the Milky Way. 


\section{DISCUSSION}

The main objective of our work has been a quantitative description of energetic protons and electrons in halos of SFGs, based on detailed modeling of their SSDs in galactic disks. We adopted an approximate diffusion solution to particle distributions in the halo, normalized at the disk boundary by using detailed results from GALPROP models for the Galaxy. Our treatment provides a realistic basis for estimating the particle radiative yields in the halo for an assumed magnetic field profile, halo gas mass, gas core radius, and diffusion coefficient. Doing so we bracket the most likely ranges of spectral and spatial profiles of halo radio, hard X-ray, and $\gamma$-ray emission; these constitute a tangible basis for assessing the detection feasibility of the integrated fluxes in these spectral bands.

Results of our calculations indicate that emission from the halo of a SFG will be comparable to or higher than that of the disk only in the hard X-ray band. This clearly is due to the relatively high fraction of secondary electrons in the halo and the uniformity of the CMB (the dominant radiation field), in sharp contrast to the steeply decreasing magnetic field and gas density that determine emission levels in the radio and $\gamma$-ray, respectively. In our 'fiducial' models the estimated 10-200 keV halo luminosities of NGC 4631 and NGC 4666 are $\sim 17$ and $\sim 26$ times higher than the respective (NT) disk luminosities. For each of these galaxies the predicted luminosities in the other three models are within a factor of two of the respective values specified in Table 2. While high, the total 10-200 keV luminosity of even the (more luminous) SB galaxy NGC 4666, $\simeq 2 \times 10^{39} \mathrm{erg} / \mathrm{s}$, is still substantially lower than stellar X-ray emission from the disk, which is dominated by high-mass X-ray binaries (but includes also significant emission from low-mass X-ray binaries and thermal emission from diffuse IS gas). The disk emission can be estimated using the observationally deduced scaling relation (Mineo et al. 2012) for the 0.5-8 keV luminosity in terms of the SFR, which for NGC 4666, yields a value that is more than a factor of 10 higher than the predicted 10-200 keV luminosity of the halo. We note that for the Galaxy, with a global SFR of $\sim 1 \mathrm{M}_{\odot} \mathrm{yr}^{-1}$, Strong et al. (2010) estimates for the (total) disk luminosities are $L(10 \mathrm{keV}-100 \mathrm{MeV}) \simeq 2.2 \times 10^{38} \mathrm{erg} / \mathrm{s}$, and $L(0.1-100 \mathrm{GeV}) \simeq 7.2 \times 10^{38}$ $\mathrm{erg} / \mathrm{s}$ for their diffusion model 2 .

Our estimates of hard X-ray luminosities of the two galaxies provide sufficient motivation for dedicated searches for halo emission by the next generation hard X-ray satellites which will likely have sufficient sensitivity and angular resolution to separate out disk and halo emission in nearby edge-on galaxies. Prime candidates for such observations are the two nearby, nearly edge-on SBGs NGC253 and M82, which at 2.5 and 3.6 Mpc, respectively, are more than twice closer than NGC 4631, and with significantly higher SF rates, are more optimal targets for mapping X-ray emission from their halos. The Fermi-LAT telescope detected emission above $200 \mathrm{MeV}$ from NGC253 and M82 (Abdo et al. 2010); the emission was determined to be non-varying and point-like. A recent ('Pass 8') analysis of 7-year Fermi-LAT data on a sample of SFGs (Rojas-Bravo and Araya 2016) resulted only in upper limits on the emission above $100 \mathrm{MeV}$. The sample includes NGC 4631 for which a $95 \%$ upper limit of $5 \times 10^{39} \mathrm{erg} / \mathrm{s}$ was set. (This upper limit is a factor $\sim 1.8$ lower than the value deduced by Ackermann et al. 2012 in their analysis of emission from SFGs in the 3-year Fermi-LAT database.) The current bound is somewhat higher than our predicted total $\gamma$-ray luminosity. Given the intense SF activity in the central disk regions in these galaxies, the measurement of high energy X-and- $\gamma$-ray emission from these closest SBGs galaxies is not surprising, and indeed was predicted based on detailed models (e.g., Goldshmidt \& Rephaeli 1995, Domingo-Santamaría \& Torres 2005; Persic, Rephaeli, \& Arieli 2008; de Cea del Pozo et al. 2009; Rephaeli, Arieli, \& Persic 2010).

In assessing realistic ranges of key parameters in the estimation of the predicted radiative yields of star forming galaxies, we note that in a recent analysis (Heesen et al. 2018) of radio emission profiles in nearby galaxies, higher values of the SFRs and mean disk magnetic fields were determined. The latter analysis is based on a different approach to modeling energetic electron and magnetic field distributions in the thin and thick disk regions; key differences between the respective approaches include particle source distribution (Gaussian in our treatment, uniform across the galactic disk but vanishing elsewhere in that of Heesen et al. ), the duration of the transport process (time integration is performed in our approach, whereas steady state is presumed in theirs), and our inclusion of radiative emission from secondary electrons which they (apparently) have not done. Nonetheless it is still relevant to assess the net effect of the higher values of these quantities on our results. For the two galaxies considered here, NGC 4631 and NGC 4666, SFRs of 2.9 and $16.2 \mathrm{M}_{\odot} \mathrm{yr}^{-1}$ were used; these are about a factor $\sim 2.2$ higher than the values (from Wiegert et al. 2015) adopted here. The SFR constitutes an overall normalization of particle densities in the disk, but since we normalize the predicted radio (surface) emissivity to the observed profiles, a different SFR would only affect this overall normalization factor. Mean magnetic field values in the disks of the two galaxies were taken to be 13.5 and $18.2 \mu \mathrm{G}$, higher by about $\sim 1.3$ than our values. With the level of the overall B-dependence of the synchrotron emissivity, this difference amounts to about the same (latter) factor. Therefore, given inherent observational uncertainties, and the fact that our calculated radiative yields (for the set of models considered here) span appreciably wider ranges than that implied by the above SFR and magnetic field values, the overall impact on our predictions is small.

The ratio of the particle energy lost by interactions in IS space to the energy injected out of the acceleration sites is referred to as the calorimetric efficiency. This fraction is known to be very large $(\sim 90 \%)$ for primary electrons, but quite low $(\sim 10 \%)$ for protons. Obviously, additional losses in the halo result in essentially negligibly small residual primary electron component in the outer halo. Of interest is mainly the total calorimetric efficiency of protons and their residual energy content

MNRAS 000, 1-12 (2019) 
in the outer halo. For the models explored here, our estimates indicate that the total calorimetric efficiencies of protons are in the range $\sim .1-4 \%$. Propagation through the halo increases the total proton energy losses by only a few percent over the losses in the disk.

An important consequence of the low calorimetric efficiency of protons diffusing out of SFGs is their accumulated density in clusters. In the central regions of rich clusters the gas density and magnetic fields are significantly enhanced with respect to their levels in IG space outside clusters, with typical values of $\mathrm{O}\left(10^{-3}\right) \mathrm{cm}^{-3}$ and $\mathrm{O}(1) \mu \mathrm{G}$, higher than corresponding values in the outer halo of a SFG that is not a cluster member. The higher concentration of SFGs in a cluster and stronger coupling of protons to the magnetized IC gas result in the creation of secondary electrons and the emission of significant radio and NT $\mathrm{X}-\&-\gamma$ emission in the central cluster region.

We have recently carried out a first detailed assessment of galactic proton and electron in IC space (Rephaeli and Sadeh 2016) based on a calculation of the particle spectro-spatial distributions assuming that electrons diffuse out of radio and SF galaxies. Given the lack of unequivocal observational evidence for appreciable proton component in the lobes of radio galaxies, we conservatively ignored the contribution of radio galaxies assuming that protons originate only in SFGs. The extended distribution of SFGs, whose relative fraction increases with distance from the cluster center, is a key feature in accounting for the large size of radio halos. Particle escape rates were approximated by scaling to the estimated rates from the Galactic disk (Strong et al. 2010), with no account taken of propagation and energy losses in the halo. This approach was applied to conditions in the Coma cluster, where the number of SFGs was estimated from the total blue luminosity of the cluster, and only the two central powerful radio galaxies were included as electron sources.

We found that for reasonable models of the gas density and magnetic field spatial profiles, the predicted profile of the combined radio emission from primary and secondary electrons is roughly consistent with that deduced from current measurements of the Coma halo. However, in this rather conservative approach (in accounting for proton and electron sources in clusters) the level of radio emission was found to be appreciably lower than the measured emission; this suggests that there could be additional particle sources, such as AGN and lower luminosity (than that of the two strong central) radio galaxies. Also calculated were the levels of NT X-ray emission, which was predicted to be mostly by Compton scattering of electrons from radio galaxies off the $\mathrm{CMB}$, and $\gamma$-ray emission from the decay of neutral pions produced in interactions of protons from SFGs with protons in IC gas. Since the predicted levels of NT X-ray and $\gamma$-ray emission from (e.g.) the Coma cluster are appreciably lower than current upper limits, only weak constraints can at present be obtained on the steady state proton and electron IC distributions.

In assessing the likelihood of additional proton component in clusters, we note that the low calorimetric efficiency of protons, which are clearly accelerated in the nuclei of radio galaxies, implies that these could be important proton sources. Some evidence for significant proton populations in the lobes of radio galaxies comes from Fermi-LAT measurements of emission above $100 \mathrm{MeV}$ from the nearby Centaurus A (Abdo et al. 2010) and Fornax A (Ackermann et al. 2016). Based on detailed models of the radio to $\gamma$ spectral energy distributions of these galaxies it was concluded that the measured $\gamma$ emission is from the $\pi^{0}$ decay (produced in p-p interactions), rather than Compton up-scattering of the radio-emitting electrons off the background optical radiation field (McKinley et al. 2015, Ackermann et al. 2016). However, at least in the case of Fornax A, it was recently shown (Persic \& Rephaeli 2019) that the optical radiation field in the lobes (which is dominated by the central host galaxy NGC 1316) is sufficiently intense to account for the Fermi-LAT measurements by Compton scattering. It remains to be seen if the measured emission from the Coma halo can be fully accounted for by protons and electrons from all $\mathrm{SF}$ and radio galaxies in the cluster. If this turns out to be unrealistic, then there would be stronger motivation to consider particle re-acceleration (in IC space) as an alternative model for maintaining the requisite level of radio-emitting electrons.

\section{ACKNOWLEDGEMENTS}

This work has been supported by a grant from JCF (San Diego, CA). The authors wish to thank Theresa Wiegert for clarifying details of the radio-map in the CHANG-ES 2015 paper, and the referee for a constructive report.

\section{REFERENCES}

Abdo A.A. et al., 2010, ApJ , 719, 1433

Ackermann M., et al., 2012, ApJ. 755, 164

Ackermann M., et al., 2016, ApJ. 826, 1

Aharonian F.A., Atoyan A.M., 1996, Astron. Astrophys. , 309, 917

Atoyan A.M., Aharonian F.A., \& Völk H.J., 1995, Phys. Rev. D. , 52, 6

Blumenthal G.R., Gould R.J., 1970, Rev. Mod. Phys. , 42, 2

de Cea del Pozo E., Torres D.F., Rodriguez Marrero A.Y., 2009, ApJ, 698, 1054

Domingo-Santamaría E., Torres D.F., 2005, A\&A, 444, 403

MNRAS 000, 1-12 (2019) 
Gilmore G., Reid N., 1983, MNRAS , 202, 1025

Goldshmidt O., Rephaeli Y., 1995, ApJ , 444, 113

Gould R.J., 1972, Physica, 60, 145

Heesen V. et al., 2018, MNRAS , 476, 158

Kelner S.R., Aharonian F.A., Bugayov V.V., 2006, Phys. Rev. D. , 74, 034018

Mannheim K., Schlickeiser R., 1994, A\&A, 286, 983

McKinley B., et al., 2015, MNRAS , 446, 3478

Mineo S., Gilfanov M., Sunyaev R., 2012, MNRAS, 419, 2095

Orlando E., 2019, Phys. Rev. D. , 99, 043007

Orlando E., Strong A., 2013, MNRAS, 436, 2127

Persic M. Rephaeli Y., Arieli Y., 2008, A\&A, 486, 143

Pfrommer C., Enßlin T.A., 2004, Astron. Astrophys. , 426, 777

Ramaty R., Lingenfelter R.E., 1966, JGR, 71, 3687

Rephaeli Y., Arieli Y., Persic M., 2010, MNRAS , 401, 473

Rephaeli Y., Sadeh S., 2016, Phys. Rev. D. , 93, 10

Rojas-Bravo, C., Araya, M., 2016, MNRAS, 463, 1068

Stein Y., 2017, Magnetic Fields and Cosmic Ray Transport in Edge-On Spiral Galaxies from the CHANG-ES Sample, Ruh-Universität Bochum

Strong A.W., Porter T.A., Digel S.W., Jóhannesson G., Martin P., Moskalenko I.V., Murphy E.J., Orlando E., 2010, Ap. J. Lett. , L58

Syrovatskii S.I., 1959, Soviet Astronomy, 3, 22

Wiegert T. et al., 2015, Astron. J. 150, 81

This paper has been typeset from a $\mathrm{T}_{\mathrm{E}} \mathrm{X} / \mathrm{L} \mathrm{T} \mathrm{E} \mathrm{X}$ file prepared by the author. 